\title{
Civilisations
}

Revue internationale d'anthropologie et de sciences

humaines

60-1|2011

Mobilisations et dynamiques identitaires en Amérique andine

\section{Jeux du corps et jeux identitaires chez les Cholas}

(La Paz, Bolivie)

\section{Laura Fléty}

\section{(2) OpenEdition}

\section{Journals}

Édition électronique

URL : http://journals.openedition.org/civilisations/2707

DOI : $10.4000 /$ civilisations. 2707

ISSN : 2032-0442

Éditeur

Institut de sociologie de l'Université Libre de Bruxelles

Édition imprimée

Date de publication : 31 décembre 2011

Pagination : 23-42

ISBN : 2-87263-035-X

ISSN : 0009-8140

Référence électronique

Laura Fléty, « Jeux du corps et jeux identitaires chez les Cholas », Civilisations [En ligne], 60-1 | 2011,

mis en ligne le 12 février 2015, consulté le 19 avril 2019. URL : http://journals.openedition.org/

civilisations/2707 ; DOI : 10.4000/civilisations.2707

(c) Tous droits réservés 


\title{
Jeux du corps et jeux identitaires chez les Cholas (La Paz, Bolivie)
}

\author{
Laura FLÉTY
}

Résumé : Cet article s'attache à comprendre la construction d'une nouvelle identité urbaine féminine, celle des "Cholas » de la ville de La Paz, en Bolivie. Ces femmes, facilement identifiables dans l'espace urbain par leurs tenues vestimentaires, participent massivement à l'une des plus grandes fêtes patronales de la ville en dansant la Morenada. Par la pratique de cette danse, les Cholas mettent en place de complexes processus d'identifications en exposant une esthétique corporelle spécifique face à des alter féminins, plus précisément face à d'autres corporéités féminines présentes dans la performance. La danse et les normes qu'elle sous-tend, devient une manière particulièrement efficace pour ces femmes de rendre visible l'idée d'un collectif mais aussi d'affirmer une identité " autre " qui combine à la fois les symboles de la société urbaine contemporaine bolivienne et des représentations indiennes et rurales.

Mots-clés : corps, Bolivie, Morenada, constructions identitaires, Cholas, La Paz, femmes.

Abstract: The aim of this article is to understand the construction of a new urban identity of women, the "Cholas" of the city of La Paz, in Bolivia. These women, that are easily identifiable in the urban space by their dress, are involved in one of the most important celebrations of the town, performing the Morenada dance. Through the practice of this dance, the Cholas build a complex identification process by exposing a specific body aesthetics, in order to conform a position regarding other women's corporealities present in the performance. The dance and the standards that support it, are an effective way for these women to make visible the idea of a community but also to assert a "different" identity that combines the symbols of bolivian urban society as well as indigenous and rural representations.

Keywords: bodies, Bolivia, Morenada, construction of identities, Cholas, La Paz, women. 
et article explore l'une des catégories de l'éventail des identités urbaines féminines de La Paz : les Cholas. Ces dernières sont principalement identifiables par leur apparence. Elles portent quotidiennement la pollera, lourde jupe colorée agrémentée de jupons, se coiffent de deux longues tresses, portent un châle et un chapeau en équilibre sur la tête. Revêtir la pollera implique pour ces femmes un positionnement identitaire manifeste puisque d'une part, elles marquent volontairement une rupture esthétique et socio-économique avec le monde rural et indien ${ }^{1}$ et que d'autre part, elles se distinguent d'une mode occidentale (tailleur, robe, pantalons) adoptée par la majorité des femmes appartenant à d'autres milieux sociaux. Une enquête ethnographique (trois longs séjours entre 2008 et 2011) menée au sein de plusieurs fraternidades ${ }^{2}$ - structures sociales dans lesquelles s'organisent et dansent les Cholas en l'honneur d'un Saint ou d'une Vierge - nous amène à nous interroger sur les nouveaux processus d'identification de ces femmes et les négociations quotidiennes à travers lesquelles ils s'élaborent. Ces ajustements identitaires ne se jouent pas uniquement au niveau des discours mais également sur le corps des acteurs et leur mise en mouvement, notamment à travers la danse. Celle-ci doit être considérée en tant qu'acte performatif qui crée des espaces dans lesquels les relations entre les participants se modifient et se négocient perpétuellement (Cánepa Koch 2001 : 18). Ainsi, le corps n'est pas simplement le reflet d'une situation vécue, il est aussi un moyen d'action sur cette situation permettant de la renforcer ou de la contrer. Ce qui nous intéresse ici n'est donc pas uniquement la qualité expressive mais la qualité génératrice du corps.

Les termes espagnols mestizo (métis) ou cholo ont désigné, depuis l'époque coloniale, les personnes nées des relations entre Espagnols et Indiens, les Cholos étant en général associés à la couche sociale inférieure des Métis. Aujourd'hui, ces termes ne renvoient plus uniquement à une origine ethnique ou à un supposé " mélange de sang " mais également à une catégorie sociale, celle d'une population urbaine en pleine croissance, issue des vagues de migrations rurales et indiennes, principalement d'origine aymara ${ }^{3}$ en ce qui concerne la ville de La Paz. Cette population émergente travaille actuellement majoritairement dans le commerce (commerce informel et micro entreprises), les activités artisanales, les transports et les emplois domestiques. Depuis une trentaine d'années, elle a acquis une forte visibilité ${ }^{4}$

1. Les paysannes portent traditionnellement des robes en toile artisanale alors que les polleras sont faites d'un tissu industriel plus coloré.

2. On appelle fraternidades des associations dont les membres participent à la fête annuelle d'une image catholique particulière et exécutent des danses en son honneur. Ces structures seraient inspirées des confréries religieuses qui se sont formées en Europe et qui ont été implantées dans le Nouveau Monde lors de la colonisation (Fléty 2006 : 76-102).

3. Les trois principaux groupes ethniques en Bolivie sont les Quechuas, les Aymaras et les Guaranís. La population indigène aymara vit principalement dans les zones situées autour du lac Titicaca; La Paz et El Alto sont les principales villes qui accueillent les migrants de ces zones rurales et des exploitations minières de la région. Le phénomène migratoire s'est fortement intensifié après la révolution nationale de 1952-53, avec la réforme agraire et les problèmes de partage des terres ainsi qu'avec le développement de l'éducation en milieu rural (Widmark $2003: 2$ ).

4. $\quad 48 \%$ des habitants de la ville de La Paz seraient aymaras, du moins d'un point de vue linguistique (Rivera 1983 : 139). 
et un poids institutionnel et politique ${ }^{5}$ croissant (universités, administrations, chaînes de télévision, etc), phénomène accentué notamment depuis l'élection en 2005 du président « indien » Evo Morales. Les Cholos constituent cependant une catégorie extrêmement hétérogène dont les limites sont difficilement saisissables (Healy et Paulson 2000 : 9) et dont les membres n'expriment pas forcément, tout du moins à travers leurs discours, un sentiment d'appartenance sociale commune. Il existe ainsi de fortes disparités économiques entre, d'une part, les groupes de migrants aymaras récemment arrivés à La Paz ou El Alto et d'autre part, une petite bourgeoisie commerçante plus aisée, également issue des flux migratoires mais installée à la Paz depuis de nombreuses années et qui correspond, de fait, aux couches supérieures de cette population chola. L'appartenance à la catégorie cholos/cholas n'efface donc jamais complètement les différences économiques internes.

Par ailleurs, comme l'indiquent Fuenzalida et Mayer, la limite entre la catégorie cholo/ chola et celle des Indiens est souvent relative, puisque un individu sera considéré comme métis (ou cholo) dans certaines situations et comme indien ${ }^{6}$ dans d'autres (1974:20). Ces appellations ou étiquettes identitaires s'avèrent donc très ambiguës et ce, tant dans une perspective synchronique que diachronique. En effet, historiquement, selon le siècle auquel on se réfère, les catégories métis ou cholo ont aussi recouvert des réalités sociales fort différentes (Barragán 1997).

Durant la période coloniale, si les Cholos incarnent un métissage biologique, ils initient également de nouvelles dynamiques socio-économiques. Installés en ville pour travailler dans le commerce, l'artisanat ou les mines, ils adoptent un mode vestimentaire espagnol et sont considérés comme des «Indiens » en pleine ascension sociale. Ce nouveau statut leur permet notamment d'éviter le paiement du tribut à la couronne espagnole. Pour l'élite locale, ils représentent alors une importante menace pour l'intégrité du système colonial (Abercrombie 1991 : 114). Au XIX ${ }^{\mathrm{e}}$ siècle, le processus de construction de la nation bolivienne, profondément marqué par les théories racistes et le darwinisme social, marginalise encore les Indiens afin de légitimer l'appropriation illégale des terres par les descendants d'Espagnols et l'utilisation d'une main d'œuvre peu coûteuse. Dans ce contexte, les Cholos cherchent à se différencier et à se distancier du monde « indien » en migrant vers la ville, en apprenant l'espagnol et en continuant de changer leurs manières de s'habiller. Par exemple, les femmes appartenant aux classes sociales inférieures non indiennes, adoptent fréquemment la pollera dès la fin du XVIII siècle, cherchant à imiter les femmes des classes supérieures qui, elles, vont alors l'abandonner (Barragán 1996 : 59).

Enfin, au cours du $\mathrm{XX}^{\mathrm{e}}$ siècle, l'image publique des Cholas revêt encore de nouvelles significations. Les femmes portant la pollera dans la classe ouvrière, organisées dans la Federación Obrera Feminina (Fédération Ouvrière Féminine), s'imposent dans l'espace public lors de grandes manifestations, revendiquant l'amélioration de leurs conditions de vie et de travail. Face à la proposition d'un État moderne et à un discours nationaliste (1952) qui impose « une culture identitaire homogène dans laquelle priment certaines représentations de la femme et du corps maternel » (Stephenson 1999 : 9-10), la pollera devient « un

5. Voir par exemple la création du parti politique «Conscience de la Patrie » (CONDEPA) fondé en 1988 et soutenu massivement par la population chola aymara. En 1989, pour la première fois dans l'histoire du pays, une femme chola est élue au poste de députée (Alenda $2000: 19$ ).

6. Dans cet article, nous utilisons indifféremment les termes indien et indigène. 
symbole visible de résistance qui illustre la lutte quotidienne de l'Indienne et de la Chola qui a délibérément refusé d'être "refaçonnée" ». (Ibid. : 5). Ces femmes continuent donc de porter la pollera et n'adoptent pas la mode vestimentaire occidentale alors que l'idée de progrès impose à cette époque l'effacement de tout signe d'indianité. Or, pour les classes supérieures, ce vêtement renvoie irrémédiablement au monde indien, c'est à dire à la saleté ${ }^{7}$ et à la pauvreté. On observe donc l'ambigüité de la position de la Chola, qui adopte la pollera pour se distinguer de l'Indienne mais qui en même temps est toujours assimilée à celle-ci par l'élite urbaine.

Ainsi, la catégorie chola paraît marquée par la tension de l'entre-deux, occupant un espace charnière entre deux mondes, deux cultures, et ce jusqu'au $\mathrm{XX}^{\mathrm{e}}$ siècle.

\section{L'émergence de la figure de la chola représente non seulement une rupture de la dualité Indiens versus Espagnols-créoles, en relation avec l'émergence de nouvelles activités économiques et sociales, mais aussi l'interférence de valeurs entre ces deux mondes et la création d'une identité conflictuelle qui porte en son sein simultanément la 'tradition' et la 'modernité'. (Barragán 1997 : 61)}

L'ambivalence du positionnement des Cholos dans le complexe ensemble ethnique des Andes, représenterait, encore aujourd'hui, une menace pour les élites politiques de La Paz, leur rappelant quotidiennement la fragilité de leurs privilèges (Lazar 2008 : 16). Par ailleurs, ce serait cet « entre deux » ethnique et politique qui aurait induit les fortes connotations négatives liées à la catégorie cholo/chola (Seligmann 1989). En effet, ce rapide panorama historique montre comment le maintien des termes cholos/cholas a toujours désigné une position sociale marginalisée dans la société urbaine, celle-ci étant occupée par différentes populations au cours du temps. Ainsi, depuis le $\mathrm{XVI}^{\mathrm{e}}$ siècle, ces termes renvoient à un contenu extrêmement péjoratif (Peredo Beltrán 2001 : 5-10) et sont, encore aujourd'hui, emprunts d'un fort stigmate social. Les Cholas vont donc rarement s'auto-désigner comme telles. Elles préfèreront dire qu'elles sont des femmes en pollera ou des Cholas Paceñas (originaire de La Paz). Le terme «paceña», utilisé dans ce contexte, renvoie à une identité locale associée à la ville de La Paz et témoigne surtout d'une forte volonté d'adhésion à un monde urbain et moderne.

De plus, on remarque que le statut cholo/chola s'inscrit plus clairement sur les vêtements et le corps des femmes (Canessa 2005, Stephenson 1999, Weismantel 2001). En effet, la différenciation ethnique et sociale, opérée par le port d'un vêtement comme la pollera, est une pratique féminine puisque les hommes n'ont pas d'équivalent aussi significatif dans leur habillement ${ }^{8}$. En ville, la visibilité des catégories cholo et chola n'est donc pas la même et la stigmatisation touche plus fortement les femmes, leur « indianité » étant plus marquée que celle des hommes (Canessa 2005, De la Cadena 1995). Cette différence en termes de genre pourrait s'expliquer par le fait que les hommes indiens, et par extension les Cholos, sont plus susceptibles d'être considérés comme apportant une contribution à la Nation par leur travail

7. Dans Gender and Modernity in Andean Bolivia (1999), Stephenson montre la manière par laquelle la mode et les pratiques d'hygiène corporelle peuvent être appréhendées comme des métaphores de l'incorporation du contrat social et symbolique dominant. Dans ce discours hégémonique, l'habillement et le corps des Indiens sont considérés, par définition, comme malpropres.

8. Les hommes adoptent soit un style « sportif » (survêtement, casquette), soit un style vestimentaire plus formel (costume, cravate). 
salarié et le service militaire, alors que les femmes sont considérées comme étant en dehors de 1'État-nation (Canessa $2005: 16$ ).

Si l'habit identifie clairement les Cholas, d'autres critères identifient les Cholos, comme l'usage de la langue aymara, la pratique d'une profession dans le secteur tertiaire, et leur inscription dans certains espaces de la ville. Par exemple à La Paz, les Cholos contemporains c'est-à-dire les Indiens aymaras ayant massivement migré vers la ville dans les années qui suivirent la réforme agraire de 1952 et leurs enfants, nés en ville - se sont principalement installés dans les quartiers périphériques de La Paz en pleine expansion (El Alto9, quartiers Gran Poder, Munaypata, rues Buenos Aires, Max Paredes et Juan Granier). Cependant, ces critères sont trop formels et trop généraux pour permettre de saisir le contenu si fluide et complexe de la catégorie cholos/cholas. Comment redéfinir alors aujourd'hui cette catégorie urbaine?

Divers auteurs ont commencé à se pencher sur les dynamiques complexes qui traversent l'espace social, ethnique et économique occupé par les actuels Cholos et Cholas de La Paz. Le travail microsociologique d'Albó, Greaves et Sandoval $(1982,1983)$ sur une population chola principalement composée de migrants aymaras de La Paz, met en lumière les différents processus qu'impliquent leur insertion dans les structures citadines : la recherche d'un nouveau statut social et économique, les relations contradictoires tissées avec parents et amis restés dans les communautés indiennes, ou encore, le surgissement d'une nouvelle culture aymara urbaine qui s'impose en ville par la magnificence et la grandeur de ses fêtes (Albó et Preiswerk 1986).

Les travaux de Peredo Beltrán (1992) et Marchand (2006, 2007) présentent un intéressant corpus de données ethnographiques concernant des commerçantes cholas qui travaillent dans les marchés de La Paz. Le premier auteur dresse un portrait psychosocial de ce qu'il présente, peut être de manière trop essentialiste, comme « l'identité de la chola du marché », une identité flexible qui intègre à la fois des éléments de la société occidentale et d'autres d'origine andine. Marchand, quant à elle, propose une fine analyse sociologique du jeu d'identifications professionnelles, ethniques et de genre de ces commerçantes ainsi que, dans une perspective politique, des multiples luttes et grèves qu'elles ont menées ces dernières années. En ce qui concerne la construction des réseaux socio-religieux, Guaygua et Castillo (2008) montrent comment cette frange de la population pratique un catholicisme populaire à la fois empreint de références indigènes aymaras et fortement influencé par les mouvements pentecôtistes. Enfin, Guaygua (2001), Barragán et Cárdenas (2009) s’intéressent à la constitution de ce qui serait une nouvelle bourgeoisie chola participant aux fêtes urbaines et activant d'importantes stratégies de différenciation afin d'affirmer un nouveau pouvoir socioéconomique et symbolique.

La réflexion qui suit s'inscrit dans la continuité de ces études récentes cherchant à appréhender les nouvelles constructions identitaires des Cholos et Cholas de La Paz. Mon propos est d'interroger certaines catégories urbaines, au féminin, en présentant une ethnographie qui met l'accent sur les «événements du corps ». Les Cholas ne se seraient pas constitués en tant que groupe ethnique et n'auraient pas acquis une auto-identification et une auto-dénomination. (Barragán 1997 : 67). Or, cette situation n’implique pas l'absence d'un

9. Depuis 1984, El Alto est officiellement une ville à part entière qui est devenue, ces dernières années, l'une des plus grandes agglomérations de Bolivie. 
sentiment collectif, sentiment qui apparaît moins dans le discours des acteurs que par le biais d'autres procédés et d'autres pratiques ${ }^{10}$ (rituels, fêtes, répétitions de danse, consommation d'alcool, cérémonies religieuses etc.). Parmi ces dernières, il s'avère que celles ayant trait au corps, celles qui le transforment, le mettent en mouvement, le donnent à voir, ou encore, le font interagir avec d'autres corps, occupent une place privilégiée dans l'expression de l'appartenance au groupe.

Cet article analysera l'une des activités collectives qui mobilise le plus d'énergie, de temps et d'argent des Cholos du quartier commerçant Gran Poder de La Paz : la danse Morenada, dont les enjeux de pouvoir et de prestige ont été mis en avant par Barragán et Cardenas (2009). La pratique de la danse, avec les normes esthétiques qu'elle sous-tend, est l'une des manières les plus efficaces permettant à ce groupe social de s'affirmer, de « faire corps » et d'exprimer l'idée d'un collectif.

Ce travail, volontairement ethnographique, est construit à partir de plusieurs entretiens réalisés auprès de femmes qui participent à l'une des plus grandes festivités religieuses de la ville, la Fiesta de Jesús del Gran Poder. Durant celle-ci, elles défilent en cortège pour danser la Morenada, danse dont elles répètent les pas et les chorégraphies pendant des mois. Ce sont des Cholas qui ont entre quarante et cinquante ans, arrivées adolescentes à La Paz pour travailler dans le commerce informel ou comme employée domestique. Ce sont également des commerçantes qui, bien que vivant dans les mêmes quartiers que les Cholas, ne portent pas la pollera ou du moins pas de manière quotidienne. Ce sont aussi de jeunes étudiantes, filles ou filleules des Cholas ou encore des filles issues de la classe moyenne urbaine et qui n'ont, hors de l'espace de la fête que peu de relations quotidiennes avec les pratiques et les espaces liés à la culture aymara urbaine des Cholos. L'observation continue des interactions entre ces femmes, lors des répétitions collectives de danse, permet de saisir le fonctionnement de certaines catégories identitaires effectives : comment dans cette danse et à travers les différents modèles corporels qu'elle expose, les Cholas se positionnent-elles par rapport à des alter féminins ? Précisons enfin que pour comprendre certaines normes corporelles, celles qui relèvent du « beau », ou simplement des attitudes gestuelles socialement partagées et validées, nous avons appris à porter la pollera et le chapeau, à nous coiffer et à pratiquer la danse Morenada pour participer aux fêtes avec les Cholas. Ceci a été la seule façon d'appréhender des représentations et pratiques du corps à peine explicitées par ailleurs. Ces dernières, peu étudiées jusqu'à présent, paraissent être un pilier fondamental de l'expression d'un Nous, difficilement identifiable par ailleurs. Comme Csordas le suggère, prenons au sérieux la proposition de Mauss dans son célèbre essai sur les techniques du corps (1950) : la culture s'ancre et prend forme dans le corps humain qui est « à la fois l'outil original par lequel les hommes façonnent le monde et à la fois la substance originale dont le monde humain est formé » (Csordas $1994: 6)$.

\section{La danse Morenada ou l'affirmation d'une esthétique urbaine}

L'une des pratiques fondamentales dans la création d'un sentiment collectif au sein de la population chola de La Paz, concerne sa participation massive aux « fêtes folkloriques et religieuses » et notamment à l'une des plus prestigieuses de La Paz : la Fiesta de Jesús del

10. Voir sur cette question Lazar 2008 : 262-263. 
Gran Poder ${ }^{11}$. Celle-ci rassemble chaque année plusieurs milliers de danseurs. À travers une construction visuelle sophistiquée, ces derniers présentent, sous forme de cortèges, différentes danses ${ }^{12}$ mettant en scène une série de personnages : animaux fantastiques, Indiens, guerriers, personnages coloniaux ou êtres de l'inframonde. Ces grandes manifestations possèdent un contenu religieux manifeste, à savoir, dans notre cas, la dévotion à la figure de Jesús del Gran Poder, mais les enjeux sociaux (hiérarchisations internes, enjeux de genre, dynamiques de compétition) s'avèrent aussi importants pour les participants que les aspects religieux (Fléty $2006: 20)$.

Si la fête mobilise un vaste spectre social, on constate que les Cholos/Cholas sont omniprésents $^{13}$. Ils exposent leurs propres codes esthétiques puisqu'ils sont les artisans (brodeurs spécialisés, couturières, cordonniers, etc.) des costumes et des masques visibles dans la fête et s'imposent aussi numériquement en tant que membres du public, musiciens ou danseurs. En outre, le secteur artisan des brodeurs cholos, installés principalement dans la zone commerçante du Gran Poder, est lié à l'émergence de cette fête religieuse. L'urbanisation de cette zone commence au début du $\mathrm{XX}^{\mathrm{e}}$ siècle grâce à l'intense activité commerciale, notamment la vente de produits de l'Altiplano. C'est en 1922 ou 1923 que naît la Fiesta de Jesús del Gran Poder, comme expression symbolique et identitaire de ce nouveau quartier. À cette époque, les brodeurs cholos dansent la Morenada et la Diablada (Danse de diables) (Albó et Preiswerk 1986). Aujourd'hui, parmi un large choix possible de danses (Diablada, Caporales, Tinku, Tobas, entre autres), les Cholos/Cholas choisissent majoritairement la Morenada, devenue ces dernières années l'une des danses centrales de la festivité. Sur une cinquantaine de groupes de danseurs qui défilent, elle est de loin la danse la plus exécutée ${ }^{14}$. C'est d'une part un véritable effet de mode, puisqu'il y a une dizaine d'années, une autre danse, la Kullawada, était à l'honneur au sein de cette population, mais d'autre part la Morenada reste, historiquement en tout cas, fortement associée au développement du corps de métier des artisans cholos.

Par ailleurs, la Morenada peut être conçue comme l'affirmation voulue d'une esthétique urbaine. Dans une dimension visuelle, l'utilisation de masques et de costumes fabriqués à partir de matériaux industriels - tels que perles, paillettes, soies, velours aux couleurs flamboyantes - caractérise cette esthétique, par opposition à celle des costumes que l'on peut voir dans les communautés indiennes, qui utilisent plus de tissus artisanaux et des matériaux de récupération. À cette somptuosité plastique correspond par ailleurs, en musique, l'utilisation de fanfares d'une grande puissance sonore qui partout en Bolivie, exécutent les musiques des Cholos à l'occasion des fêtes patronales ou des mariages (Martínez 2001 : 171). La

11. Pour une histoire et analyse de la fête de Jesús del Gran Poder, voir Albó et Preiswerk (1986) et Guss (2006).

12. Une quinzaine de danses, appelées « danses folkloriques », sont exécutées lors de la fête de Jesús del Gran Poder. Certaines, telles la Morenada, la Diablada ou la danse Caporales, sont actuellement omniprésentes dans toutes les fêtes patronales de Bolivie. Elles participent d'un phénomène d'uniformisation des traditions régionales qui donne lieu à la création d'une culture nationale et urbaine (Fléty 2006 : 46).

13. C'est l'une des particularités de la fête de Jesús del Gran Poder puisque dans d'autres fêtes comme le Carnaval d'Oruro ou celle de la Vierge Guadalupe à Sucre, d'autres secteurs sociaux se mobilisent (Fléty 2006).

14. Constat établi à partir des programmes du défilé des groupes de danseurs pour la fête du Gran Poder 2009 et 2010 . 
Morenada compte également un bloc complet de danseuses cholas portant une pollera brodée, c'est-à-dire une version luxueuse de leur habit traditionnel quotidien. C'est là encore un référent direct à l'urbanité puisque, comme nous l'avons déjà dit, les polleras des paysannes indiennes sont visuellement très différentes. Enfin, le dispositif chorégraphique de la Morenada consiste en un grand assemblage de blocs et de files de danseurs minutieusement organisés (guides de danse, sous-guides). Cette manière d'organiser l'espace dansé, partagée avec les autres danses de la fête, peut être qualifiée de spécifiquement urbaine puisque les danses des communautés indiennes présentent d'autres types de dispositifs.

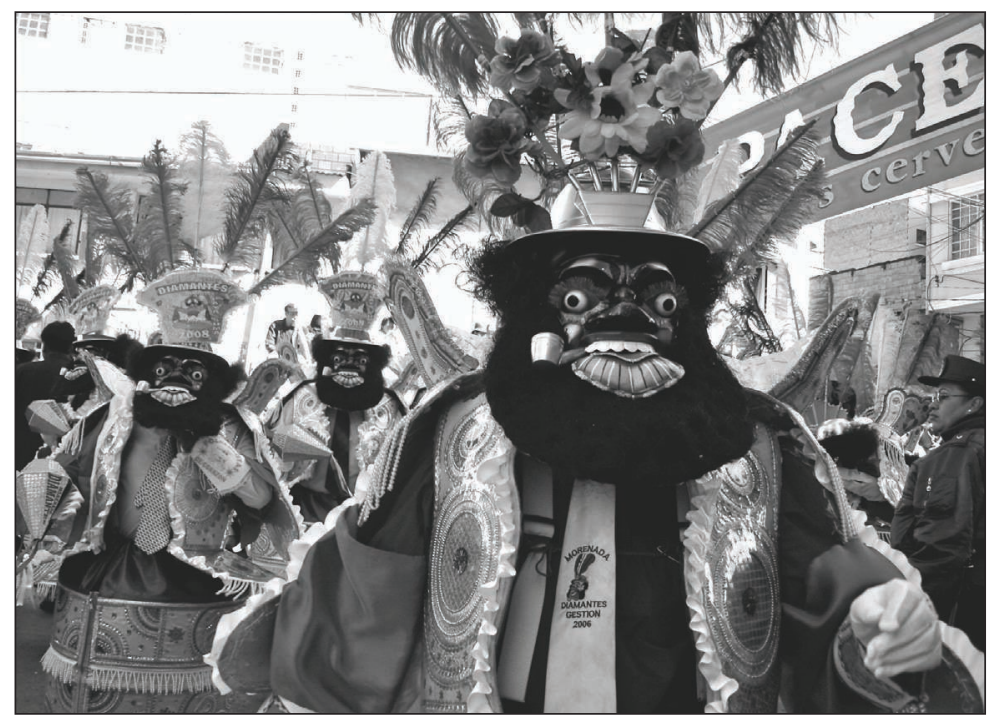

Photo 1

Personnage du moreno de la Morenada Diamantes (Fête du Gran Poder, la Paz 2008).

(C) Laura Fléty.

À une autre échelle, la Moredana participe également à la création d'une culture nationale dans laquelle le monde urbain se reconnaît : la danse est exécutée à l'occasion de divers événements officiels, visible sur les calendriers, affiches et posters des ambassades et immanquablement présente dans les clips touristiques diffusés en boucle dans les aéroports. Cette association entre identité nationale et identité métisse correspond au surgissement, assez tardif en Bolivie, d'une conscience nationale ${ }^{15}$. C'est avec la révolution de 1952, menée par le Mouvement National Révolutionnaire (MNR), que se met en place pour la première fois un véritable projet gouvernemental dont l'ambition est de créer une " nouvelle nation » bolivienne (Burgos 2005). Cette nouvelle conscience nationale se cristallise autour du concept d'une « Bolivie métisse » censée inclure tous les habitants du territoire. La nationalisation du pays se fait à travers la mise en exergue d'un « peuple bolivien » qui triomphe des traditions particularistes : ainsi, tout indigène peut devenir métis en abandonnant son identité indienne

15. En 1989, Calderon et Laserna affirmaient que la question nationale n'était pas encore résolue et continuait de représenter un problème majeur en Bolivie (Calderon et Laserna 1989 : 275). 
pour devenir citoyen de la Nation (Urioste 2005 : 64-65). Actuellement, le gouvernement d'Evo Morales, en place depuis 2005, cherche à construire un tout autre projet de citoyenneté qui aurait comme ambition de "décoloniser» la Bolivie (Lazar 2010 : 186). Selon la constitution de 2009, la Bolivie est dorénavant un État plurinational qui cherche à consolider la pluralité identitaire du pays et n'oblige plus ses citoyens à abandonner leur appartenance ethnique pour appartenir à la Nation.

L'origine et les significations attachées à la Morenada sont loin d'être consensuelles. Certains auteurs affirment qu'elle représente la souffrance des esclaves noirs pendant l'époque coloniale (Fortún 1976) ${ }^{16}$. Mais pour les danseurs d'aujourd'hui cette évocation reste lointaine même si certains éléments de la danse, tels les masques représentant des visages noirs, ou les personnages masculins appelés morenos, « ceux qui ont la peau foncée », font indiscutablement allusion à l'univers de l'esclavage. Les recherches actuelles montrent cependant qu'il est peu probable que cette danse ait été exécutée par les esclaves africains, arrivés en Bolivie dès le XVI ${ }^{\mathrm{e}}$ siècle. L'historienne María Luisa Soux (2003) indique que cette danse aurait plutôt comme origine les danses de Maures et Chrétiens, exécutées en Espagne et dans toute l'Amérique coloniale.

Les personnages féminins, intégrés seulement il y a une trentaine d'années n'ont, quant à eux, aucun rapport avec cette représentation de l'esclavage. Il en existe deux grandes sortes : les cholas que j'ai déjà mentionnées, puis les chinas, jeunes danseuses portant des bottes à hauts talons et une très courte jupe, dont l'esthétique est proche de celle des majorettes américaines. Il s'agit pour ces dernières, d'une esthétique qui apparaît, au premier abord, en contradiction totale avec le modèle féminin aymara incarné par les Cholas habillées de leurs longues et volumineuses polleras.

Des distinctions sociologiques sont repérables entre les danseuses de Morenada:

- Tout d'abord, les chinas, qui dansent dans des files séparées, sont de jeunes étudiantes de la classe moyenne.

- Ensuite, le bloc de cholas se compose de femmes qui sont réellement des Cholas dans la vie quotidienne et de femmes appelées «de vestido », « en robe $»^{17}$. Ces dernières s'habillent couramment à la mode occidentale urbaine mais ont pris, pour la danse, l'habillement des Cholas.

- Enfin, les Cholas « anciennes » (antiguas) dansent à part et sont des femmes de la classe moyenne/aisée en costume de chola du début du $\mathrm{XX}^{\mathrm{e}}$ siècle. Elles cherchent à se détacher des danseuses du bloc des cholas en utilisant des accessoires d'une autre époque qui leur confèrent une distinction sociale et culturelle (Barragán et Cardenas 2009 : 363).

Ainsi, autant les cholas antiguas que les femmes « en robe » représentent une altérité, alors que les Cholas « authentiques » sont, elles, en autoreprésentation.

16. Plusieurs hypothèses sur l'origine très controversée de la Morenada sont proposées dans l'ouvrage de Cuba et Flores (2007).

17. L'expression «de vestido, « en robe » désigne, par extension, les femmes habillées à la mode urbaine occidentale. 


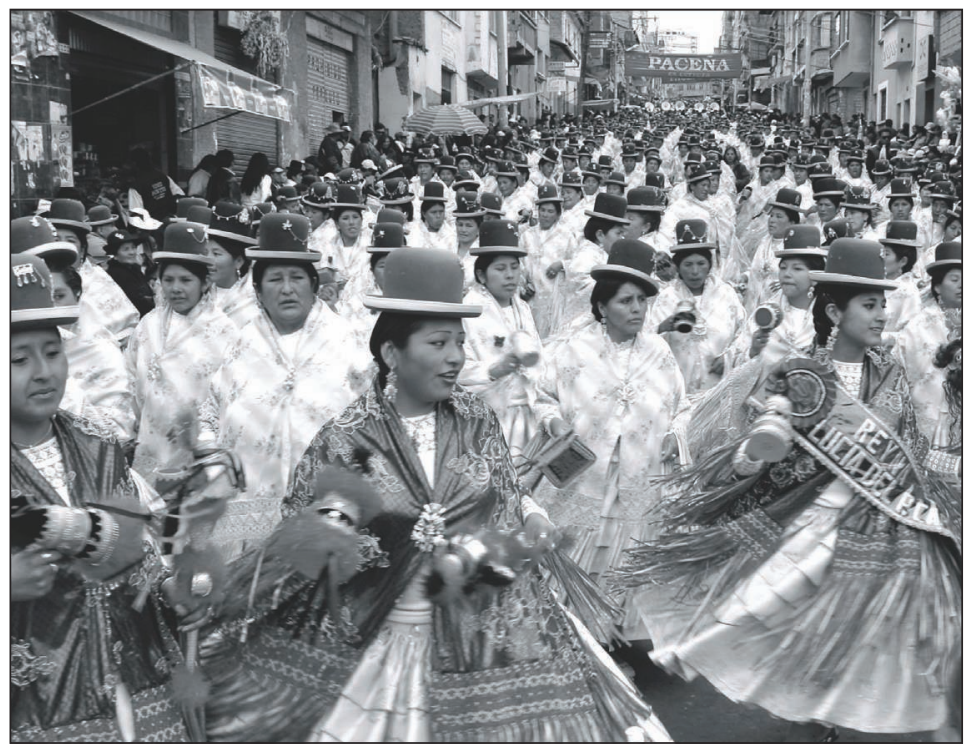

Photo 2

Défilé des Cholas de la Morenada Rosas de Viacha (Fête du Gran Poder, La Paz, 2008).

(C) Laura Fléty.

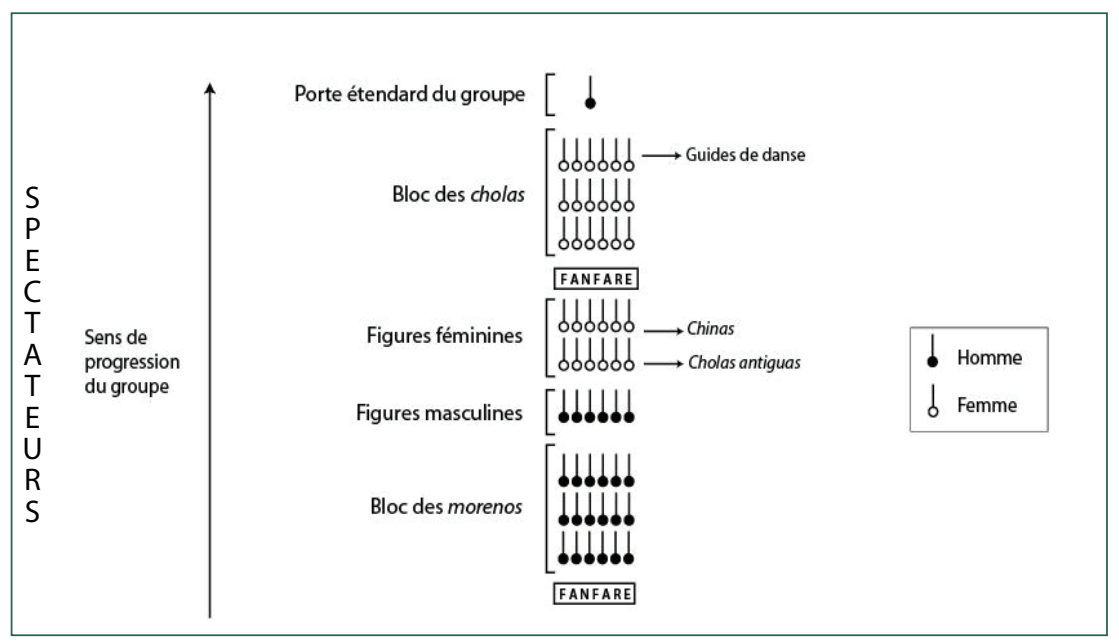

Schéma 1

Exemple du dispositif (simplifié) d'une Morenada. 


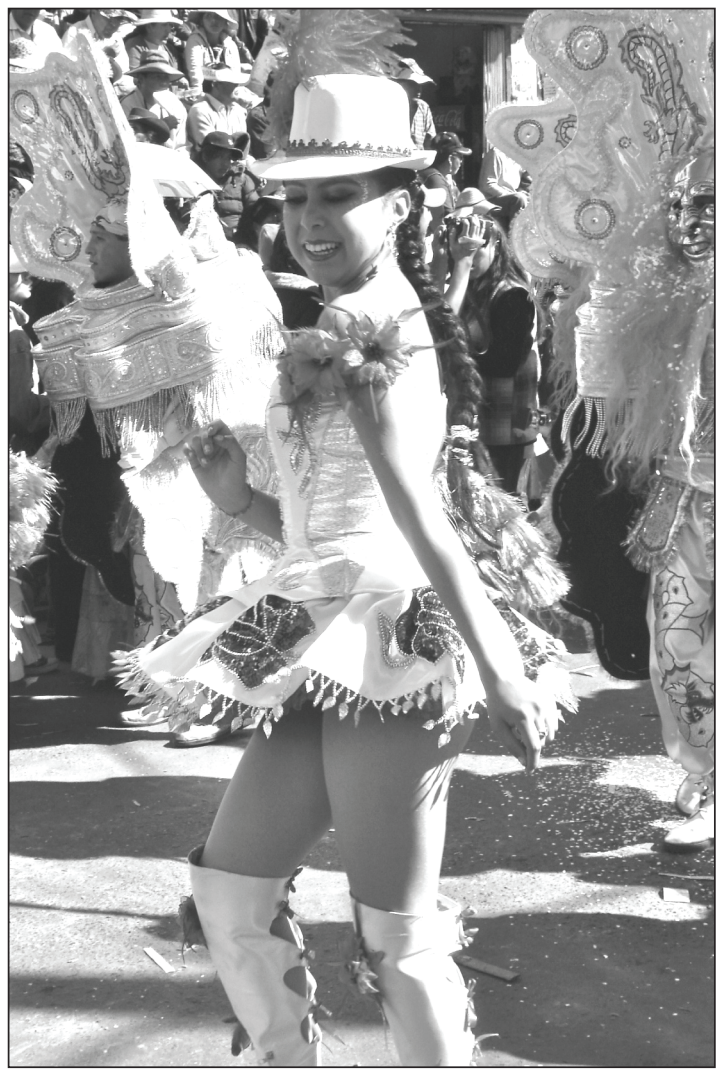

Photo 3

Danseuse china de la Morenada Los Catedráticos (Fête du Gran Poder, La Paz, 2008).

(C) Laura Fléty.

\section{La mise en scène de deux modèles féminins}

Au sein de la Morenada, les danseuses en pollera et les chinas ne sont pas de la même génération. Les premières sont en majorité des femmes mariées, ayant déjà des enfants et dont le mari danse également dans la Morenada, avec le bloc des figures masculines en fin de cortège. Les seules jeunes filles qui participent dans le bloc de cholas (bloc composé parfois de plus de 500 danseuses), sont les filles ou les filleules des Cholas. En revanche, les chinas sont beaucoup moins nombreuses à danser. Leurs files séparées se composent de cinq ou six danseuses. Lors des répétitions collectives, les chinas sont en petit groupe et restent souvent à part. Leurs attitudes corporelles mettent en valeur une coquetterie assumée selon les canons de beauté véhiculés par les médias et les concours de Miss : assises, elles croisent souvent les jambes, posent leurs mains sur les hanches et arborent de larges sourires. Elles portent des vêtements moulants qui soulignent les courbes du corps, chaussent de hauts talons et ont un maquillage très prononcé. Lors des chorégraphies, les bras sont particulièrement mobilisés, la gestuelle des mains est précise et délicate, le but étant de ressembler, selon leurs termes, à 
de « petites poupées ». Enfin, durant la danse, elles soulèvent régulièrement le tissu de leurs courtes jupes exposant ainsi le haut de leurs cuisses. Il faut noter que ce type de mouvement s'inscrit dans un répertoire gestuel féminin et urbain plus large, qui n'est pas spécifique à la Morenada, puisqu'on le retrouve par exemple chez les danseuses caporales (Fléty 2006 : 61), autre danse exécutée pendant la fête par des jeunes filles issues généralement de la classe moyenne ou aisée. Ainsi les chinas, comme d'autres danseuses avec qui elles partagent des codes esthétiques et chorégraphiques similaires, affichent des normes corporelles qui sont perçues comme « modernes », « non indiennes » et valorisent l'exposition du corps, particulièrement les jambes, aux yeux du public.

Au contraire, la pollera couvre les jambes des Cholas, les nombreux jupons et le large châle masquent les formes du corps. Quand María - une Chola de 53 ans, vendeuse de meubles décrit la manière adéquate de s'habiller pour aller danser aux répétitions de Morenada, elle utilise fréquemment un vocabulaire lié à la dissimulation. Elle explique qu'il faut toujours bien se couvrir le corps, savoir le dissimuler " il faut être bien cachée et savoir se couvrir $\gg^{18}$ (entretien réalisé en 2009). Or, il ne s'agit là ni de timidité ni d'une volonté de s'effacer. S'il faut effectivement couvrir son corps, il doit l'être avec des tissus éclatants. Les cheveux sont recouverts mais avec un chapeau orné de bijoux. Les épaules, à l'inverse des chinas, ne sont pas dénudées mais habillées d'un châle brodé, perlé ou pailleté. En ce sens, entre les deux esthétiques féminines, on constate à la fois la présence d'une claire opposition exposition/dissimulation du corps - et d'une certaine continuité : autant les chinas que les cholas affichent un goût prononcé, pendant le temps de la fête, pour l'ostentatoire.

Lors des mouvements dansés, les Cholas avancent d'un pas lent, balançant délicatement les bras. Les mouvements sont peu expansifs, plutôt contrôlés et l'intention est de ne pas dévoiler certaines parties du corps.

Je n'aime pas montrer mes jambes, j'ai honte, si je veux danser en bougeant plus alors je me fais faire plus de jupons. Mais attention, pas des jupons larges qui se soulèvent, des jupons plus étroits, sinon les gens critiquent et disent : " que c'est laid la manière dont bouge cette femme, regardez comme elle se montre alors qu'elle n'est pas une femme en vestido ", ils vont critiquer, ils vont dire que je suis coquette. (Verónica, Chola de 40 ans, vendeuse de vêtements ; entretien 2009)

À travers leurs gestes, les Cholas expriment une certaine pudeur corporelle, il ne faut pas « en faire trop », la modération étant valorisée tant dans la danse que dans la gestuelle que l'on observe lors des répétitions et des fêtes de quartier. Assises bien droites, toujours avec le chapeau en équilibre sur la tête, les mouvements sont souvent parcimonieux et modérés, les Cholas cherchant avant tout, selon leurs termes, à être " élégantes ». Elles se moquent d'ailleurs des chinas qu'elles appellent « las peladas », « celles qui sont à poil ». Pour autant, la présence des chinas dans la Morenada n'est pas remise en question par les Cholas qui les considèrent davantage comme une "valeur ajoutée », participant au succès du groupe et suscitant l'admiration du public (les groupes de Morenada sollicitent régulièrement des actrices ou des mannequins pour danser).

Cependant les Cholas ne souhaitent généralement pas que leurs filles dansent en china. Certaines pensent que cela peut être source de mauvaise réputation (« une fille trop coquette 
ce n'est pas bien vu, ça attire trop de problèmes, trop d'ennuis, les gens parlent ! » dit Victoria, une Chola de quarante ans), mais la majorité explique qu'il s'agit surtout d'un statut qui manque de prestige :

Je n'aurais pas voulu que ma fille danse de china, cholita c'est mieux, c'est bien vu, c'est comme ça que l'on dépense bien, parce qu'il faut lui acheter ses bijoux, son chapeau, le tissu pour faire la pollera, les chinas, pfff, elles ne font que louer leur costume, ça n'a aucun coût, tout le monde aurait ri! (Adela, Chola de 42 ans, épicière ; entretien 2008)

En effet, danser dans le bloc des femmes en pollera implique de fortes dépenses (la jupe, les bijoux et le châle) que beaucoup de femmes métisses ne peuvent assumer. Ainsi, danser pour la fête du Gran Poder est une manière d'afficher une stabilité financière et un pouvoir économique, que celui-ci soit réel ou fictif ${ }^{19}$. Par ailleurs, les propos d'Adela indiquent l'émergence de nouvelles représentations liées au port de la pollera. Généralement associée à une population précaire (pauvre et largement analphabète), la jupe devient, dans l'espace rituel de la fête, un objet suscitant l'admiration. La qualité et la finesse du tissu, choisi et cousu avec soin, font alors de cette pièce de l'habillement un symbole central du prestige social.

On voit à travers ces exemples que si les modèles féminins, incarnés par la china et la chola, paraissent au premier abord complètement opposés, ils ne le sont pourtant pas à tous les niveaux et ne sont pas vécus uniquement sur le mode de l'opposition. En examinant de près ces deux modèles, on s'aperçoit de la présence d'un certain nombre de continuités : goût pour l'ostentatoire, recherche d'élégance, importance du détail et de la combinaison des couleurs, uniformité complète avec les autres femmes de son groupe, désir de prestige, qu'il soit économique pour les Cholas et plutôt basé sur des qualités d'ordre physique pour les chinas. Ces continuités sont en fait nécessaires pour afficher une appartenance au même ensemble esthétique que constitue la danse Morenada.

\section{Les danseuses en pollera, relations intergénérationnelles}

Dans le bloc des cholas se produisent à la fois des femmes qui sont des Cholas dans la vie de tous les jours et qui portent quotidiennement la pollera et à la fois des femmes de vestido qui le temps de la fête, s'habillent comme les Cholas. Celles-ci sont les filles ou filleules des Cholas ainsi que des parentes, voisines ou amies avec qui elles peuvent avoir des relations de parenté spirituelle (relations de compérage et parrainage). Si pour les Cholas, danser la Morenada consiste à exposer des codes esthétiques qui leur sont familiers, pour les deuxièmes il s'agit de s'approprier ces codes : il faut apprendre à se tresser, à mettre les jupons dans le bon ordre, à « bien » porter la jupe, le châle et les bijoux, ou encore à tenir le chapeau sur la tête sans qu'il n'en tombe. Ce qui relève de ce que l'on pense être des « détails » vestimentaires engage en fait des différences notables dans les comportements corporels, la posture et la démarche. Ces comportements affirment une " manière d'être » qui, au-delà d'être spécifique à chacun, marque l'appartenance au groupe social. Par exemple, le port, en équilibre, du chapeau melon, nécessite un mouvement d'impulsion vers le haut du corps et un

19. Certaines femmes font de grands sacrifices personnels (économies, emprunts, travail supplémentaire) afin de pouvoir danser pour le défilé. 
relâchement au niveau des hanches qui donne cet aspect « digne " au niveau du port de tête et la souplesse du bassin, caractéristiques des Cholas qui marchent en ville.

L'apprentissage de ces techniques du corps est l'objet d'un long processus de transmission entre les mères et leurs filles ou leurs filleules. À travers une série de conseils tels que : «Baisse la pollera, on voit trop tes jambes ", « cache plus tes tresses sous le châle ", « remonte tes jupons », les Cholas transmettent à leurs filles des valeurs à la fois esthétiques et morales. Lors des répétitions de danse, les mères restent en permanence aux côtés des jeunes filles. Par mimétisme, ces dernières acquièrent un type de corporéité socialement valorisé par le groupe : une retenue et un contrôle permanent du corps. Cette attitude est constante que ce soit dans le rapport au sexe opposé, dans les pratiques sociales partagées comme la forte consommation d'alcool ou encore, dans la danse même. En effet, il est courant, pendant la performance, d'entendre le public critiquer l'aspect « éteint » des Cholas, interprété souvent comme un manque d'enthousiasme. Elles sont en effet moins démonstratives et nettement moins emphatiques dans leurs mouvements que les chinas par exemple. Au niveau du visage, l'expression est en général plutôt figée et affiche une certaine impassibilité. Il n'y a pas d'intention expressive explicite. Si la danse nécessite un effort physique important et constant (le défilé dure parfois jusqu'à six heures), la fatigue n'est jamais affichée en public. Tout passe par un subtil jeu du contrôle de soi et de son ressenti corporel. On peut alors émettre comme hypothèse que ce comportement physique participe à ce que l'on pourrait désigner comme une corporéité « chola». Notons en passant la continuité avec un type de corporéité également présent dans les communautés indiennes :

[...] gestes indigènes et gestes métis ont en commun une caractéristique : ils ne sont jamais expressifs, ne cherchent pas à communiquer des affects, des émotions. Les sentiments pouvant traverser les musiciens, joie, douleur, nostalgie, émotion religieuse... ne s'exposent pas au regard des autres. (Martínez 2000 : 172)

Enfin, la transformation ponctuelle des filles « en robe » pour devenir des filles en pollera induit une identification commune entre les générations des mères et des filles. C'est avec fierté et émotion que certaines mères préparent leurs filles pour aller danser la Morenada. Ainsi Victoria s'étonne lorsqu'elle voit pour la première fois sa fille, vêtue de l'une de ses pollera : «Je ne savais pas que tu étais belle comme ça, en cholita tu es tellement différente. Je pensais que tu ne me ressemblais pas du tout, mais maintenant tes tantes disent que l'on est identiques ! » (Victoria, Chola d'environ 40 ans, femme au foyer ; entretien 2008). Ou encore, Dolores me confie : «Ma fille, la pauvre, elle est bien moche, je lui dis de ne pas sortir dans la rue avec autant de maquillage! Mais dans les fêtes, on dirait une autre personne, la pollera lui va si bien! » (Dolores, Chola de 52 ans, couturière; entretien 2010). Toutefois, si les jeunes filles intègrent les valeurs morales et esthétiques transmises par leurs mères, elles réinventent et se réapproprient leurs codes. Par exemple, pour la danse, elles se maquillent et mettent du vernis sur les ongles alors que les Cholas ne le font pas. Elles affichent ainsi une coquetterie plus marquée et montrent une volonté de différenciation avec la génération plus âgée : « Nous dansons dans le bloc des polleras, mais nous sommes jeunes, coquettes et modernes! » affirme Lydia, 25 ans, fille de Chola (entretien 2010). Cet engouement des filles de Cholas pour danser la Morenada est un phénomène très récent puisque la Morenada était jusqu'alors plutôt considérée par la jeunesse comme une danse « des vieux » (Barragán et Cardenas 2009 : 369). 
L'identification commune mère-fille et la transmission de codes corporels spécifiques révèlent une nouvelle dynamique pouvant apparaître comme contradictoire avec la volonté, parfois affirmée par les Cholas, d'avoir des filles qui ne leur ressemblent pas. En effet, elles refusent souvent que leurs filles portent la pollera afin que ces dernières ne souffrent plus la stigmatisation sociale qu'elles-mêmes ont supportée. Elles désirent également que leurs filles s'intègrent dans d'autres secteurs et réseaux socio économiques de la société urbaine et ce par le biais des études. Comme Marchand le dit explicitement :

L'ascension sociale, tant désirée par les Cholas, s'accompagne généralement d'un changement vestimentaire de leurs filles ; un changement qui suit toujours le même trajet : être de vestido alors que sa mère est de pollera. Ce trajet signifie donc une rupture par rapport à la tradition vestimentaire. Être de vestido signifie que l'on grimpe dans l'échelle sociale, c'est le signe d'une mobilité sociale ascendante intergénérationnelle. (Marchand $2001: 217$ )

Rappelons qu'au sein de la fête, la pollera est un symbole de prestige et de succès économique et c'est donc uniquement sous cette condition que les Cholas acceptent, pendant le temps du rituel, que leurs filles s'identifient à leur catégorie sociale.

\section{Normes du corps et distinctions identitaires}

Le jour du défilé dansé, les blocs de cholas exhibent une machine chorégraphique synchronisée et imposante : elles exécutent simultanément les mêmes mouvements accompagnés de chants. Au niveau de l'habillement coloré et brillant, l'uniformisation est complète. Mais, cette unité et homogénéité mise en avant durant la performance, cache, de fait, les différences de statut entre les danseuses « de pollera » et « de vestido ». Ces dernières ont d'ailleurs été désignées péjorativement par les journalistes comme les « fausses cholitas » ou les «transformers ». Cependant, si les Cholas, dans leurs discours, marquent une nette différence entre nous et elles, aucun jugement de valeur négatif n'est émis. Elles ne perdent néanmoins jamais l'occasion de montrer qu'elles sont les connaisseuses légitimes de la mode vestimentaire chola. Par exemple, la veille du défilé, lorsque toutes les danseuses vont récupérer leurs tenues (jupe et châle) chez la couturière, ce sont les Cholas qui se posent en expertes. Elles examinent avec soin, des heures durant, la qualité et la longueur du tissu, la finesse des broderies et exposent à voix haute leurs critiques, à côté des femmes « de vestido ». Ces dernières écoutent avec attention ces commentaires, afin d'apprendre à juger le travail des couturières et à ne pas se laisser duper.

En outre, les différences entre les deux catégories sont explicites tant dans les mouvements dansés que dans les gestes ordinaires produits lors des moments de socialisation (les répétitions collectives qui précèdent la fête). Le tableau qui suit restitue les propos d'Adela, (Chola d'environ une cinquantaine d'années, vendeuse de chapeaux ; entretien 2010) qui évoque les importants contrastes existants entre les normes corporelles des femmes en pollera et des femmes en vestido.

Les propos d'Adela esquissent les contours d'une identité collective chola qui s'exprime par les « événements » du corps et les valeurs qu'elle sous tend : la honte de montrer son corps, la pudeur relative à certaines parties de celui-ci (cheveux, jambes) ou encore la valorisation d'un certain type de corporéité comme la corpulence. Notons que ce dernier point ne s'exprime pas à travers des gestes mais concerne plus la recherche d'un " état du corps » particulier. Pour être « bien » habillée lors de la performance, les Cholas créent du volume 
et se grossissent en revêtant des couches successives : collants, série de jupons, pollera, haut à manche longue, gilet, châle, bijoux, chapeau. Par exemple, quand María (Chola de 53 ans, vendeuse de meubles) m'habille en chola pour aller danser, elle s'afflige : « Moi aussi quand je suis trop maigre, la pollera ne me va plus, elle tombe, ça ne va pas! Mais toi, tu ne peux pas sortir comme ça, les gens vont rire, il faut que tu manges, où est ta graisse ? ». La graisse est une substance particulièrement valorisée, les Cholas apprécient généralement les fritures, le porc et autres viandes grasses (nourritures que l'on mange notamment avant de danser) et considèrent qu'avoir une bonne réserve de graisse est signe de bonne santé. Or, les pratiques alimentaires des Cholas qui dansent la Morenada rappellent les importantes disparités économiques qui les séparent des indiennes récemment immigrées en ville, qui ne portent pas le même type de pollera et qui sont associées à la pauvreté et à la mendicité.

\begin{tabular}{|c|c|}
\hline Femmes " de vestido" & Femmes " de pollera " \\
\hline $\begin{array}{l}\text { "Les femmes en vestido soulèvent leurs jupes, } \\
\text { elles n'ont pas honte de montrer leurs jambes " }\end{array}$ & $\begin{array}{l}\text { "Il y a beaucoup de honte, il y a de la honte de } \\
\text { bouger, honte de montrer nos jambes» }\end{array}$ \\
\hline $\begin{array}{l}\text { «Les femmes en vestido ne savent pas se tresser, } \\
\text { elles font leurs tresses trop hautes, elles n'ont } \\
\text { pas les cheveux assez longs » }\end{array}$ & $\begin{array}{l}\text { "Quand ce sont des femmes en pollera, elles } \\
\text { sont plus naturelles, elles ont leurs cheveux } \\
\text { longs naturels et elles n'ont pas besoin de se } \\
\text { recoiffer tout le temps" }\end{array}$ \\
\hline $\begin{array}{l}\text { "Les chapeaux tombent, elles ne savent pas } \\
\text { 'manier' le chapeau, elles sont obligées de le } \\
\text { faire tenir avec des barrettes" }\end{array}$ & $\begin{array}{l}\text { «Je porte le chapeau en faisant n'importe quel } \\
\text { mouvement, s'il tombe un peu je fais un petit } \\
\text { coup de tête comme ça, il faut bien le placer, en } \\
\text { mettant le petit trait de l'étiquette derrière » }\end{array}$ \\
\hline «Elles bougent beaucoup » & $\begin{array}{l}\text { "Les femmes en pollera bougent peu et } \\
\text { doucement, elles sont plus 'éteintes'» }\end{array}$ \\
\hline «Elles sont plus fines et font des régimes » & $\begin{array}{l}\text { «Nous, celles qui mettons la pollera, nous } \\
\text { aimons être bien à l'aise, un peu grosse, sinon } \\
\text { ce n'est pas beau » }\end{array}$ \\
\hline $\begin{array}{l}\text { «Elles se recoiffent dans la rue, devant tout le } \\
\text { monde et elles n'ont pas honte » }\end{array}$ & $\begin{array}{l}\text { "Nous n'allons jamais dans la rue sans être } \\
\text { bien coiffées, bien arrangées et soignées. Les } \\
\text { cheveux ne doivent pas se voir, c'est notre } \\
\text { coutume. Nous devons savoir nous couvrir, si } \\
\text { les cheveux dépassent, il faut relever le châle } \\
\text { dessus pour les cacher». }\end{array}$ \\
\hline
\end{tabular}

Tableau 1

Différences relatives aux normes corporelles des femmes en vestido versus pollera selon Adela (Chola d'environ une cinquantaine d'années, vendeuse de chapeaux ; entretien 2010).

Enfin, le tableau ci-dessus, réalisé à partir du discours d'Adela sur les normes du corps émergeant au sein d'une pratique chorégraphique, rend bien compte de l'écart qui peut exister entre les pratiques corporelles des Cholas et celles des femmes en vestido. Cependant, 
ce serait se méprendre que d'envisager ces pratiques comme exclusives à chacune de ces catégories féminines. À mon sens, ces pratiques s'inscrivent plutôt dans un même continuum identitaire : en ville, une femme adopte donc une gestualité spécifique qui l'identifie comme plus ou moins Chola. Rosa, petite fille de Chola, six ans, illustre parfaitement cette idée quand elle annonce « Ma grand mère est Chola, ma mère un peu et moi je ne sais pas encore !» (2011).

\section{Conclusion}

Ainsi, les aspects gestuels et corporels expriment d'une manière ou d'une autre et à des degrés divers, un positionnement des Cholas par rapport au monde indigène et au monde métis avec lequel elles s'identifient et qu'elles assimilent simultanément à la modernité. C'est à travers l'agencement d'éléments provenant d'univers culturels différents que se définira et se maintiendra une identité collective qui ne peut exister que dans un contexte d'opposition et de relativité. La formation de cette nouvelle identité collective chola se construit donc comme « un système d'écarts et de différences par rapport à des « autres » significatifs dans un contexte historique et social déterminé » (Poutignat et Streiff-Fenart 1995 : 192). On peut alors comprendre la présence, au sein de la danse, c'est-à-dire à l'intérieur d'une même unité spatio-temporelle, la coexistence de plusieurs modèles féminins, incarnés par les Cholas, les chinas ou encore les femmes «de vestido » qui s'habillent en pollera pour la danse. Or, ces catégories ne sont pas si étanches puisque nous avons vu, à travers l'exemple des filles de Cholas, que ces dernières oscillaient entre des codes esthétiques propres à leurs mères et des codes plus proches des chinas. Ainsi, être chola paraît être plus une affaire de degrés que de nature. Au lieu de considérer uniquement des univers esthétiques qui s'opposent, il s'agit à mon sens d'une construction collective dans laquelle les Cholas se re-positionnent sans cesse, dans un complexe jeu identitaire qui combine les symboles de la société urbaine contemporaine avec des représentations indiennes et rurales. En ce sens, les Cholas ne sont pas uniquement des intermédiaires entre la " modernité » et la « tradition », ou entre l' « urbain » et le « rural», elles sont les fondatrices d'une identité autre. Ces ajustements identitaires sont étroitement liés au phénomène d'embodiment (dans le sens de Csordas 1994) puisque ce sont bien le corps et la production chorégraphique qui permettent aux Cholas leurs prises de position originales dans un espace socio-culturel, ethnique et économique complexe.

\section{Références citées}

Abercrombie, Thomas, 1991. « To Be Indian, to Be Bolivian : 'Ethnic' and 'National' Discourses of Identity », in Greg Urban and Joel Sherzer (eds), Nation-States and Indians in Latin America, 95-130. Texas : University of Texas Press.

Albó, Xavier, Tomás Greaves et Godofredo Sandoval, 1982a. Chukiyawu. La cara aymara de la Paz. I : El Paso a la ciudad. La Paz : CIPCA.

--_, 1982b. Chukiyawu. La cara aymara de la Paz. II : La odisea de buscar pega. La Paz : CIPCA. 1983. Chukiyawu. La cara aymara de la Paz. III : Cabalgando entre dos mundos. La Paz : CIPCA.

Albó, Xavier et Matías Preiswerk, 1986. Los señores del Gran Poder. La Paz : Centro de Teología Popular.

AlEnda, Stéphanie, 2000. « La résurgence du populisme en Bolivie : "conscience de la patrie" ou la construction de nouvelles identités urbaines dans un contexte compétitif ». Bulletin de l'Institut Français d'Etudes Andines 30 (1) : 1-26. 
Barragán, Rossana, 1996. « Los Múltiples Rostros y Disputas por el Ser Mestizo », in Alison Spedding (ed.), Seminario Mestizaje : Ilusiones y Realidades, 63-106. La Paz : MUSEF.

--_-, 1997. « Entre polleras, ñañacas, y lliqllas. Los mestizos y cholas en la conformación de la "Tercera República" », in Henrique Urbano (ed.), Tradición y modernidad en los Andes, 43-73. Cusco : CBC.

Barragán, Rossana et Cleverth Cardenas, 2009. Gran Poder : la Morenada. Fiesta Popular Paceña, 3. La Paz : IEB.

Burgos, Elizabeth, 2005. « Bolivia o la pasión nacional », Nuevo Mundo Mundos Nuevos, 5, mis en ligne le 31 janvier 2005, disponible sur : http://nuevomundo.revues.org/document35.html

CAlderon, Fernando et Roberto Laserna, 1989. « Nación, Estado y movimientos sociales regionales en Bolivia (1917-1983) », in D. Camacho et R. Menjívar (eds), Los movimientos populares en América latina, 274-307. México : Siglo XXI, UNU.

CÁnepa Koch, Gisela, 2001. Identidades representadas : performance, experiencia y memoria en los andes, Perú. Lima : Centro de Etnomusicologia del Instituto Riva-Aguero de la Pontificia Universidad Catolica del Perú.

CAnessa, Andrew, 2005. «Introduction », in Andrew Canessa (ed.), Natives making nation. Gender, Indigeneity, and the State in the Andes, 3-32. Tucson: The University of Arizona Press.

Csordas, Thomas, 1994. Embodiment and experience : the existential ground of culture and self. Cambridge : Cambridge University Press.

Cuba, Simón et Hugo Flores (eds), 2007. Boliviana 100\% Paceña : la Morenada. La Paz : UMSA.

DE LA CADENA, Marisol, 1995. «Women are more Indian : ethnicity and gender in a community near Cuzco », in Brooke Larson, Olivia Harris and Enrique Tandeter (eds), Etnicity, Markets and Migrations in the Andes : At the cross roads of History and Anthropology. Durkham : Durkham University Press.

Flety, Laura, 2006. Ethnographie d'une danse urbaine de Bolivie : Les Sambos Caporales dans la fiesta de Guadalupe (Sucre). Université de Paris X-Nanterre (Mémoire de Master 1 en Ethnologie).

Fortún, Julia Elena, 1976. « Panorama del folklore boliviano », in Hugo Boero Rojo (ed.), Enciclopedia-Guía. Bolivia Mágica, 117-141. La Paz : Los Amigos del Libro.

Fuenzalida, Fernando et Enrique Mayer, 1974. El Perú de las tres razas. New York : Insituto de las Naciones Unidas para formación profesional e investigaciones.

GuAYgua Germán, 2001. Las estrategias de la diferencia : construcción de identidades urbanas populares en la festivida del Gran Poder. La Paz : IDIS-UMSA.

Guaygua, Germán et Beatriz Castillo, 2008. Identidades y Religión. Fiesta, culto y ritual en la construcción de redes sociales en la ciudad de El Alto. La Paz : ISEAT.

Guss, David, 2006. « The Gran Poder and the Reconquest of La Paz », Journal of Latin American Anthropology $11(2): 294-328$.

Healy, Kevin et Susan Paulson, 2000. Political Economies of Identity : Bolivia 1952-1998. Journal of Latin American Anthropology 5 (2) : 2-29.

LazAr, Sian, 2008. El Alto, rebel city : self and citizenship in Andean Bolivia. Durham : Duke University Press. 2010. "Schooling and Critical Citizenship : Pedagogies and Political Agency in El Alto, Bolivia », Anthropology \& Education Quarterly 41 (2) : 181-205.

Marchand, Véronique, 2001. «Les Cholas des marchés de La Paz : une approche interactionniste », Cahiers des Amériques latines $36: 207-224$.

, 2006. Organisations et protestations des commerçantes en Bolivie. Paris : L'Harmattan. 
----, 2007. «L'ethnicité revendiquée : entre union et distinction. Essai sur les autodésignations des commerçantes de La Paz », in Jean-Pierre Lavaud et Isabelle Daillant (éds), La catégorisation ethnique en Bolivie, 125150. Paris : L'Harmattan.

Martínez, Rosalía, 2001. "Autour du geste musical andin », Cahiers de Musiques traditionnelles, « le geste musical» $14: 167-180$.

Paredes-Candia, Antonio, 1992. La Chola Boliviana. La Paz : Isla.

Peredo Beltrán, Elizabeth, 2001 [1992]. Recoveras de los Andes. La Paz : Ildis-Tahipamu.

Poutignat, Philippe et Jocelyne Streiff-Fenart, 1995. Théories de l'ethnicité. Paris : Puf.

RiverA, Silvia, 1983. « Luchas campesinas contemporáneas en Bolivia : el movimiento katarista, 1970-1980 », in René Zavaleta Mercado (ed.), Bolivia, Hoy, 129-168. México : Siglo XXI.

Seligmann, Linda, 1989. " To be in Between : The Cholas as Market Women », Comparative Studies in Society and History 31 (4) : 61-69.

Soux, María Luisa, 2003. «El culto al apóstol Santiago en Guaqui, las danzas de moros y cristianos y el origen de la Morenada. Una Hipótesis de trabajo », Anales de la Reunión anual de Etnología. La Paz : MUSEF.

Stephenson, Marcia, 1999. Gender and Modernity in Andean Bolivia. Texas : University of Texas Press.

Urioste, Blas, 2005. « ¿De la Nación-minoría, al Estado Plurinacional o Nación Multicultural? » Lazos 7 : 61-69.

Weismantel, Mary, 2001. Cholas and Pishtacos : stories of race and sex in the Andes. Chicago : University of Chicago Press.

Widmark, Charlotta, 2003. To make Do in the City. Social identities and Cultural Transformations among Aymara Speakers in La Paz. Uppsala : Uppsala University Library. 